\title{
Pulling Digital Data from a Smart Object: Implementing the PullMe-Paradigm with a Mobile Phone
}

\author{
Steve Hinske \\ Institute for Pervasive Computing, ETH Zürich \\ Clausiusstr. 59, 8092 Zürich, Switzerland \\ steve.hinske@inf.ethz.ch
}

\begin{abstract}
This paper presents the PullMe paradigm, an interaction technique for easily initializing and confirming the transmission of digital data using a mobile phone. The main idea and benefit is the substitution of less feasible techniques such as manually selecting or confirming a service (e.g., by entering a password) with a simple hand gesture. We describe a prototypical implementation that utilizes an acceleration sensor and radio frequency identification (RFID) technology integrated into a mobile phone. The transmission of data is realized using Bluetooth. We furthermore discuss how near-field communication (NFC) is likely to enable interaction patterns like the PullMe paradigm in the near future.
\end{abstract}

Keywords: PullMe paradigm, human-computer interaction, HCI, semantic mapping of physical and virtual action, mobile phone, acceleration sensor, radio frequency identification, RFID, near-field communication, NFC.

\section{Introduction}

This paper presents the concept of the PullMe paradigm and describes a prototype that implements it. The PullMe paradigm is a novel interaction technique for easily downloading digital data using a mobile phone.

The main idea and major benefit is to substitute traditional selection and confirmation techniques, which are often rather intricate and time consuming (e.g., manually selecting services and typing in keys or passwords), for a simple hand gesture. Analogous to the TouchMe and PointMe paradigms [1, 2], where a device is used to touch an object or to point at it, respectively, the PullMe paradigm realizes the idea of triggering an action by pulling the device away from an object.

For a better understanding, visualize the following scenario: you want to see a movie and you are waiting for your friend in front of a cinema. Since your friend is late and you have not yet decided which movie you are going to see, you intend to use the time of waiting to pick a movie already. But before you can actually choose one, you need to have more information about the movies currently running, for example by viewing the trailers of the movies in question.

For this reason, you go to one of the advertising movie posters at the cinema, get your mobile phone out of the pocket, and you hold it close to the poster. After a few 

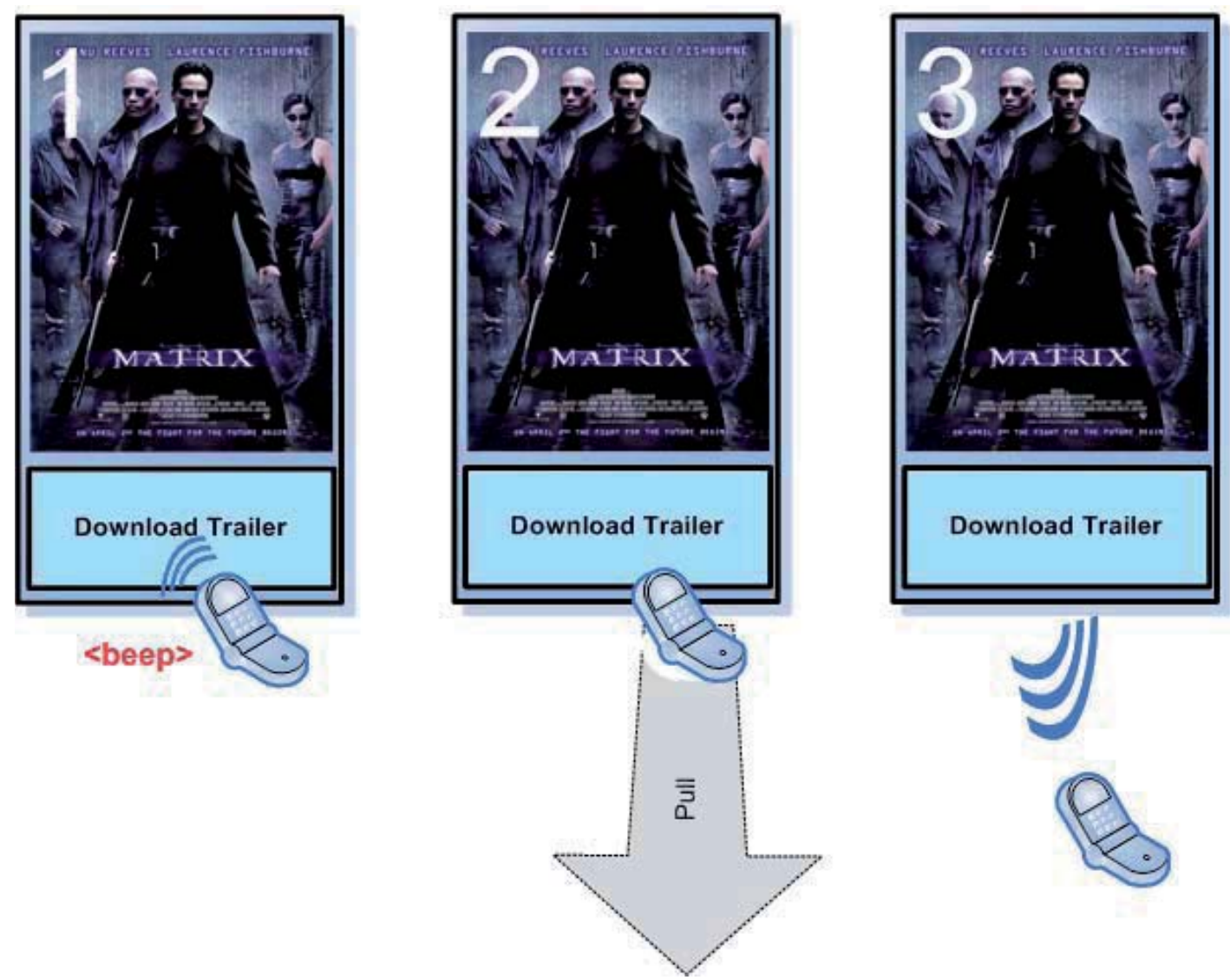

Fig. 1. Downloading a movie trailer from a poster: first, the user holds the mobile phone close to a designated area ('Download Trailer') on the poster. An acoustic 'beep' acknowledges the reciprocal detection of the mobile phone and the device integrated in the poster which offers the download of the trailer (1). The user then physically pulls the device away from the poster, thus confirming that she or he really wants to download the trailer; a virtual connection between the two devices is established subsequently (2). Finally, the trailer is then transmitted to the mobile phone using the already established connection (3).

seconds, your mobile phone gives you a short signal (e.g., an acoustic 'beep'). You then make a swift pull-away move and, subsequently, triggered by this simple gesture, a trailer of this movie is transmitted to your phone (see Fig. 1).

The scenario demonstrates the principle of the PullMe paradigm: by physically pulling away a device (e.g., the mobile phone) from a smart object (e.g. a technically enhanced movie poster or billboard), the user initiates a virtual pull: the transfer of digital data. In the case of the movie trailer, the "pulled" data is information about the actual movie. It would also be possible, however, to provide other digital assets, for example, a coupon for a free drink "pulled" from an advertisement billboard.

The remainder of the paper is structured as follows: first, we introduce the conceptual approach of the PullMe paradigm, including the communication technologies that we use. Second, we discuss the technical realization of a prototype with the focus of this section being on the employed hardware. Third, we conclude this paper with a summary and planned future work. 


\section{Conceptual Approach}

The PullMe paradigm is an interaction pattern that meets two important requirements of human-computer interaction: it is easy-to-use and quite intuitive. By replacing cumbersome menu navigation and manual configuration of services with two simple moves (holding the mobile phone in close proximity to a billboard and then move it away with a swift pull-away gesture, respectively), we can increase the usability significantly. The PullMe paradigm is also quite intuitive due to the semantic mapping of the physical and the virtual action (i.e., the virtual pull of data is triggered by and corresponds to the physical pull of the device (see Fig. 2)).

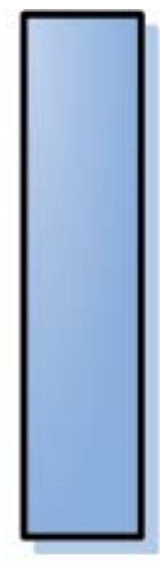

Billboard

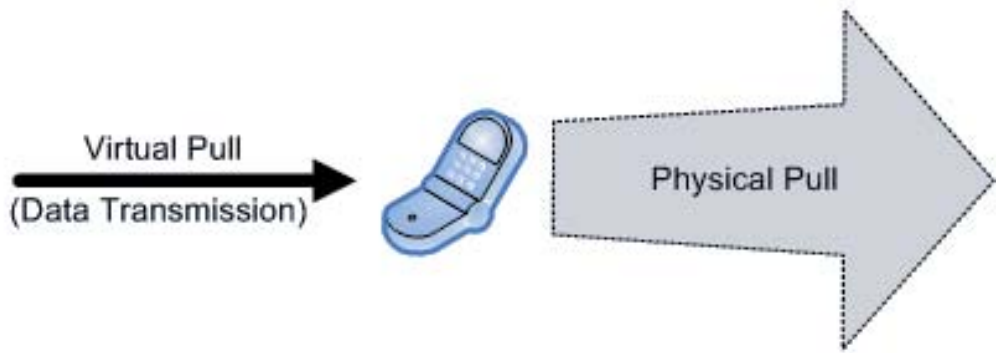

Mobile Phone

Fig. 2. The semantic mapping between physical and virtual pull action: the virtual pull (i.e., the actual transmission of data from the billboard to the mobile phone) corresponds with and is triggered by the physical pull (i.e., the physical move of the mobile phone away from the billboard)

Thus, we have two main components: on the one hand, we must recognize the pull move which is realized with an acceleration sensor attached to the mobile phone; and, on the other hand, we have to ensure that the devices detect each other beforehand. The principle of bringing two devices closely together in order to initiate a connection is mainly based on the Touch and Connect paradigm, one of the four possible application categories for near-field communication (NFC) presented by Philips [3]. But since currently available mobile phones do not provide full NFC functionality, we have to build our own device that "simulates" this functionality.

We use radio frequency identification (RFID) technology to enable the devices to unambiguously detect each other. To this end, both the mobile phone and the billboard are equipped with an RFID reader and RFID tag(s) which allows reciprocal detection and the subsequent establishment of an initial connection between them. At this moment, however, there is no data transmitted yet.

The transmission must be explicitly authorized by the user doing a swift pull-away move, which is recognized by the aforementioned integrated acceleration sensor. After a successful confirmation, the data is transmitted via Bluetooth (although it is also possible to use other communication technologies such as general packet radio service (GPRS) or universal mobile telecommunications system (UMTS)). 


\section{Technical Realization}

To realize the PullMe paradigm, the mobile phone must be equipped with an RFID tag, an RFID reader module, and an acceleration sensor. We attach a custom-built RFID reader module (a BTnode [4] that operates a Skyetek M1-mini RFID reader [5]; the BTnode is connected via Bluetooth to the mobile phone) to a Nokia 6630. In order to detect the pull-away move, we use a SparkFun WiTilt v2.5 [6], a wireless 2dimensional accelerometer that also uses Bluetooth to transmit its gathered acceleration data to the mobile phone.

An application aggregates the data from the RFID reader and the acceleration sensors, and implements the protocol of the PullMe paradigm: based on the reciprocal detection, an initial connection is established, and, if a pull-away move has been recognized successfully (meaning the transmission has been confirmed), the data is eventually transmitted from the billboard to the mobile phone (see Fig. 3).

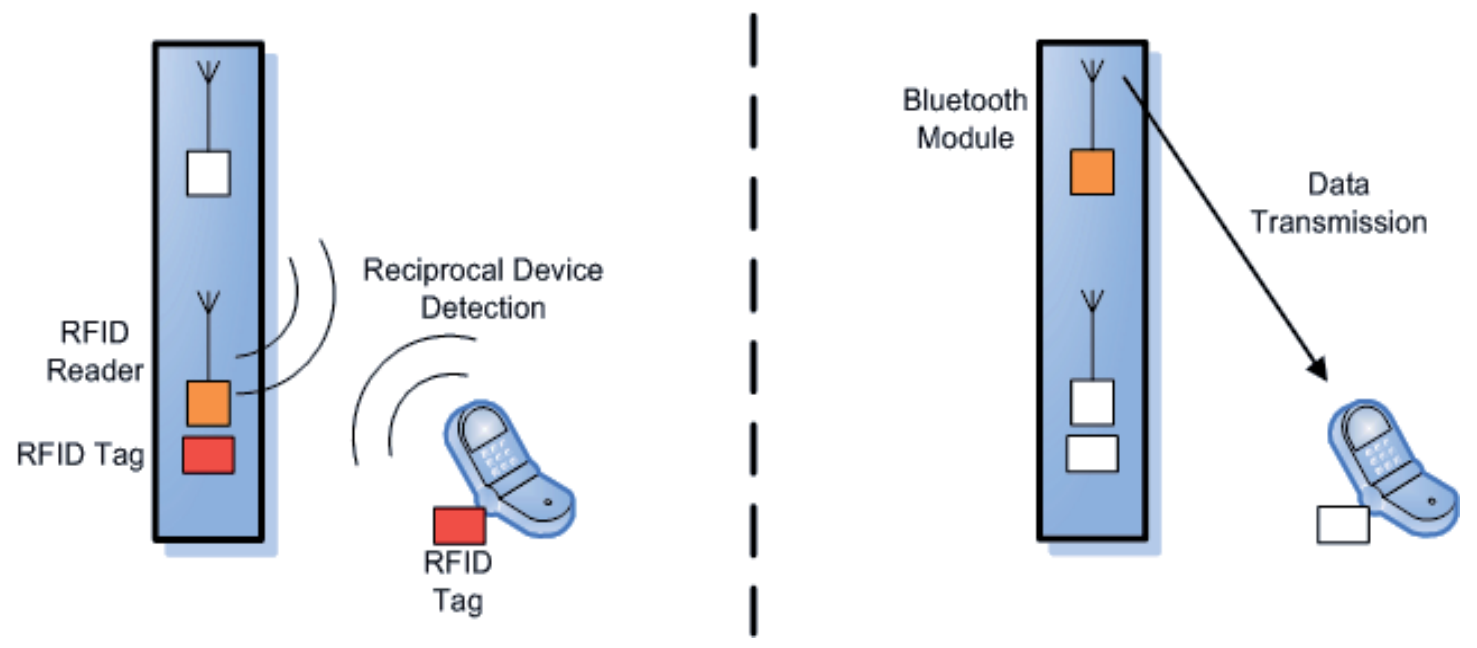

Fig. 3. Reciprocal detection via RFID (left) and the subsequent transmission of data via Bluetooth (right): first, the billboard and the mobile phone detect each other using RFID technology (i.e., both devices are equipped with RFID readers and RFID tags). This leads to the establishment of a Bluetooth connection between the devices. The second step consists of transmitting data from the billboard device to the mobile phone via Bluetooth after the transmission has been confirmed by the user using the PullMe paradigm.

This requires the billboard also to be equipped with a Bluetooth device, an RFID reader, and several RFID tags (this is necessary to cover the complete 'download area'). The tags of the billboard are read by the mobile phone, while the billboard simultaneously detects the mobile phone.

As mentioned before, the reciprocal detection is the initial step of the data transfer, but the consequential action (e.g., downloading the movie trailer) has to be confirmed by the user beforehand using a simple gesture. The main advantage is that the user neither needs to use the keyboard of the mobile phone nor has to navigate through the menu; as a matter of fact, she or he does not even have to pay attention to the mobile phone itself. 


\section{Conclusion}

In this paper we presented the PullMe paradigm, a new interaction paradigm that augments a physical move by coupling it to a "virtual move" (i.e., a transmission). In comparison with navigating through a menu, the pulling-away move is in many cases much more convenient for confirming such a transmission, thus, making the PullMe paradigm an intuitive and easy-to-use addition to human-computer interaction.

We presented a prototypical system that implements this paradigm and demonstrates the general idea based on a real world example. We intend to improve the system, especially from a technological point of view. However, it is very likely that the self-constructed prototype with the externally attached RFID reader and acceleration sensor is to be replaced by mobile phones with integrated near-field communication technology and built-in acceleration sensors in the near future.

In this context we might also extend the prototype to being able to detect visual codes using the built-in camera. This approach would result in an alternative to the RFID/NFC-based detection, but the confirmation of the data transmission, however, remains unaltered. Another point is that, instead of using Bluetooth, it might be desirable and probably more feasible to use other transmission technologies, mainly GPRS and UMTS. The advantage in this case is that the data does not have to be stored in the poster, but can be accessed on the Internet using web links.

We furthermore intend to conduct a user study to evaluate the presented interaction pattern. The aim of the planned study is to evaluate the significance and intuitiveness of the PullMe paradigm compared to other (traditional) means of retrieving digital data. The evaluation should then disclose on how easy to use and on how intuitive our approach really is.

\section{References}

1. Pohjanheimo, L., Keränen, H., Ailisto, H.: Implementing touchme paradigm with a mobile phone. In: Proceedings of the joint conference on Smart objects and ambient intelligence: innovative context-aware services: usages and technologies (2005)

2. Rukzio, E., Leichtenstern, K., Callaghan, V., Schmidt, A., Holleis, P., Chin, J.: An Experimental Comparison of Physical Mobile Interaction Techniques: Touching, Pointing and Scanning. In: Dourish, P., Friday, A. (eds.) UbiComp 2006. LNCS, vol. 4206, Springer, Heidelberg (2006)

3. Huang, J.: Access Just a Touch Away, (16.02.2007) Available at: http://www.iss.nus.edu.sg/ iss/article_display.jsp?artid=1218

4. BTnode Homepage (16.02.2007) Available at: http://www.btnode.ethz.ch/

5. Skyetek Homepage (16.02.2007) Available at: http://www.skyetek.com

6. SparkFun Homepage (16.02.2007) Available at: http://www.sparkfun.com/commerce/ product_info.php?products_id=254 